\title{
Effect of Graphitic Nanomaterials on Thermal, Mechanical and Morphological Properties of Polypropylene Nanocomposites
}

ISSN: 2576-8840

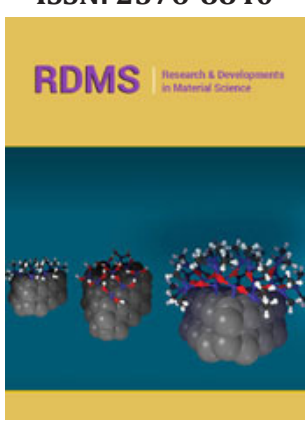

*Corresponding author: Satyendra Mishra, University Institute of Chemical Technology, Kavayitri Bahinabai Chaudhari, North Maharashtra University, Umavi Nagar, Jalgaon 425001, Maharashtra, India

Submission: 監 August 15, 2019

Published: 海August 28, 2019

Volume 11 - Issue 4

How to cite this article: Chetan R Mahajan and Satyendra Mishra. Effect of Graphitic Nanomaterials on Thermal, Mechanical and Morphological Properties of Polypropylene Nanocomposites. Res Dev Material Sci. 11(4).RDMS.000766.2019.

DOI: 10.31031/RDMS.2019.11.000766

Copyright@ Satyendra Mishra, This article is distributed under the terms of the Creative Commons Attribution 4.0 International License, which permits unrestricted use and redistribution provided that the original author and source are credited.

\section{Chetan R Mahajan and Satyendra Mishra*}

University Institute of Chemical Technology, India

\begin{abstract}
Graphene oxide (GO): Polypropylene (PP) and reduced graphene oxide (RGO): PP nanocomposites were prepared by reinforce separately by adding $0.05 \mathrm{wt} . \%, 0.1 \mathrm{wt} . \%, 0.2 \mathrm{wt} . \%, 0.5 \mathrm{wt} . \%, 1.0 \mathrm{wt} . \%$ and $2.0 \mathrm{wt} . \%$ in the PP by solution blend method. GO, RGO and their PP composites were characterized by Fourier Transform Infrared Spectroscopy (FTIR), X-ray diffraction (XRD), Field emission scanning electron microscopy (FESEM) and Transmission electron microscopy (TEM). FTIR showed that GO consists of functional moieties, which are absent in RGO. XRD patterns illustrated exfoliation of graphite to GO with an increased interlayer spacing of $1.03 \mathrm{~nm}$, which decreased in RGO (interlayer spacing $0.37 \mathrm{~nm}$ ). FESEM images showed separate sheets of GO; and RGO with mostly wrapped surface. TGA results illustrated the improvement in thermal stability of GOPP and RGOPP nanocomposites with respect to virgin PP. The tensile strength of nanocomposites of GOPP and RGOPP was improved and recorded as $44 \mathrm{MPa}$ and 42 MPa respectively over the virgin PP (28 MPa). Dynamic mechanical analysis (DMA) of GOPP and RGOPP nanocomposites also showed improvement in the storage modulus compared to neat PP. Morphological images indicated smoothing of the surface due to uniform dispersion and chain holding capacity of GO and RGO. The overall study also shows that GOPP registered better thermal and mechanical properties as compared to RGOPP.
\end{abstract}

Keywords: Polypropylene; Graphene oxide; Reduce graphene oxide; Thermal properties; Mechanical properties

\section{Introduction}

Graphitic nanomaterials such as GO, RGO and carbon nanotubes (CNT) have attracted great interest in recent years because of their various applications such as mechanical, thermal, optical, and electrical properties [1-6]. PP is one of the commercial importance polyolefins because of its cost-effectiveness as well as intrinsic properties of low density, high stiffness, good tensile strength and inertness toward acids, alkalis and solvents [7]. PP has also been used in a wide range of applications including packaging [8], textiles [9], automotive components $[10,11]$, aerospace $[12,13]$, home applications [14], construction $[15,16]$ etc. as per say; therefore, the improvement in the properties of PP is further needed. Researchers around the world tried various nanofillers such as MMT [17], nTiO 2 [18,19], nBaCO ${ }_{3}$ [20] $\mathrm{CaCO}_{3}$ [21], nano polystyrene [22], Tea Dust /GO [23], graphene [24,25] and CNT [26] to improve thermal and mechanical properties of PP matrix. Some of the researchers used functionalization of graphene using 4,4'-diphenylmethane diisocyanate (MDI) and stearic acid to improve thermal and mechanical properties of PP [24]. Ryu [27] have done the functionalization of GO by alkylamine, and studied the crystallization, mechanical and electrical properties of isotactic polypropylene nanocomposites. Polypropylene composites of alkylamine-modified GO were prepared through a melt blending technique using maleic anhydride-grafted PP as a compatibilizer. Significant improvements were observed in melt crystallization characteristics, mechanical and electrical properties upon loading alkyl amine-modified GO in PP due to strong interfacial interactions between the polymer and modified GO.

Yang et al. [28] studied the antioxidative effect of chemically reduced graphene oxide (RGO) on the thermal-oxidative stability of polypropylene (PP) was evaluated by thermogravimetric analysis and differential scanning calorimetry. They concluded that the acceptor-like electronic property afforded by the long-conjugated $\mathrm{C}$-C bonds and the barrier effect of rGO were responsible for the improved thermal-oxidation stability of PP. 
Valdes et al. [29] prepared PP/GO nanocomposites using PP grafted with amine-alcohol (PPgDMAE) as compatibilizer by two different methods. Maleic anhydride grafted PP (PPgMA) was reacted with 2-[2-(dimethylamine)-ethoxy] ethanol (DMAE) in the melt for forming amine-alcohol functionalized polypropylene (PPgDMAE). The loading of GO enhanced the mechanical, properties attributing strong interfacial interactions between GO and PPgDMAE. A significant improvement in mechanical thermal stability and electrical properties was observed when nanocomposites were prepared by the solution blending method compared with melt mixing method.

Wang et al. [30] prepared GO/short carbon fiber (SCF)/PP composite via extrusion compounding and injection molding techniques. The multiscale and synergistic effects of GO-SCF/ GO-PP showed the extensive potential on improving mechanical and thermal performance for various fields. Miao et al. [31] used PP-g-MAH as a compatibilizer for GO: PP composites. Composites containing GO contents of $0.0-5.0 \mathrm{wt} \%$ were prepared using a melt-blending method. The interfacial bonding properties, thermal properties, mechanical properties and morphological characteristics of the composite fibers were studied with GO content as the independent variable. Mantia et al. [32] studied the effect of the graphene nanoplatelets $(\mathrm{GnP})$ on the morphology and mechanical properties of polypropylene/GnP nanocomposites. The presence of the GnP magnifies the effect of elongation flow. The elastic modulus and the tensile strength of nanocomposites increases with the orientation more than pure matrix as well as the elongation at break increases at low values of the draw ratio for the nanocomposites, while, the matrix shows the expected decrease in deformability. The improvement in elongation at break was due to increase of contact surface between matrix and dispersed phase. The GnP contact area was responsible for these behaviors since the larger contact area improves the transfer of the stress from matrix to GnP. The present study is focused for an improvement in thermal, mechanical and morphological properties of PP with the incorporation of GO and RGO.

\section{Materials and Methods}

Graphite powder was procured from S.D fine chemicals, Mumbai India. Sulfuric Acid $\left(\mathrm{H}_{2} \mathrm{SO}_{4}\right)$ 98\%, Ortho-Phosphoric Acid $\left(\mathrm{H}_{3} \mathrm{PO}_{4}\right)$ 88\%, Potassium Per magnate $\left(\mathrm{KMnO}_{4}\right)$, Hydrogen Peroxide $\left(\mathrm{H}_{2} \mathrm{O}_{2}\right) 30 \%$, Hydrazine Hydrate $\left(\mathrm{H}_{6} \mathrm{~N}_{2} \mathrm{O}\right) 80 \%$ and Tetra Hydro Furan (THF) were purchased from Merk specialty Pvt. Ltd, (Mumbai, India). Toluene $\left(\mathrm{C}_{7} \mathrm{H}_{8}\right)$ was purchased from Rankem Pvt Ltd. Mumbai. Polypropylene (ICO Polymers-ICORENE ${ }^{\circledR} 4014$ Icorene $^{\circledR}$ PPCO14/RM) was procured free of cost from Heera polymers Jalgaon.

\section{Preparation of graphene oxide}

GO was synthesized from graphite powder by Improved Hummer's method as reported elsewhere [8,33-36]. Briefly, 5g of graphite powder was added to the mixture of concentrated $\mathrm{H}_{2} \mathrm{SO}_{4}$ $(600 \mathrm{ml})$ and $\mathrm{H}_{3} \mathrm{PO}_{4}(66.66 \mathrm{ml}) .30 \mathrm{~g}$ of $\mathrm{KMnO}_{4}$ was added gradually with stirring. This mixture was cooled using ice cooled water so that the temperature of the mixture was maintained below $20{ }^{\circ} \mathrm{C}$. Further, the mixture was stirred at $50{ }^{\circ} \mathrm{C}$ for $12 \mathrm{~h}$ and then terminated by adding $200 \mathrm{ml}$ of ice followed by $5 \mathrm{ml}$ of $30 \% \mathrm{H}_{2} \mathrm{O}_{2}$ solution. The mixture was filtered through polyester fiber cloth (pore size $=0.45 \mu \mathrm{m}$ ). The filtrate was centrifuged at $4000 \mathrm{rpm}$ for $20 \mathrm{~min}$, and the supernatant was discarded. The remaining solid material was washed in succession with $200 \mathrm{ml}$ of water, $200 \mathrm{ml}$ of $30 \% \mathrm{HCl}$ followed by $200 \mathrm{ml}$ of ethanol. The washed material was coagulated with $200 \mathrm{ml}$ of ether, and the resulting suspension was filtered through a polyester fiber cloth. The solid obtained was vacuum-dried at room temperature for $12 \mathrm{~h}$.

\section{Preparation of reduced graphene oxide}

The reduced graphene oxide was prepared by chemical reduction method [35,37-39]. 900mg of GO, was dispersed in $900 \mathrm{ml}$ of distilled water using bath sonicator for $1 \mathrm{~h}$, which resulted in clear dispersion with no visible particulate matter or sedimentation at the bottom. $9 \mathrm{~mL}$ of Hydrazine hydrate $\left(\mathrm{H}_{6} \mathrm{~N}_{2} \mathrm{O}\right)$ was added to this dispersion as a reducing agent and stirred for $24 \mathrm{~h}$ at $100{ }^{\circ} \mathrm{C}$ in an oil bath. Reaction mixture completely turned from brown to black in color indicating a reduction of GO. The product was isolated by filtration, washed sucessively with water and ethanol, and dried in a vacuum at $35^{\circ} \mathrm{C}$ for $12 \mathrm{~h}$ to obtain RGO.

\section{Preparation of graphitic nanomaterials based polypro- pylene nanocomposites}

The solutions were prepared as wt. \% basis of $0.05 w t . \%$, 0.1wt.\%, 0.2wt \%, 0.5wt.\% 1wt.\% and $2 w t . \%$ of GO to PP. $100 \mathrm{~g}$ of PP was dissolved in $500 \mathrm{~mL}$ of toluene at $110^{\circ} \mathrm{C}-120^{\circ} \mathrm{C}$ under constant stirring. Simultaneously, the colloidal solution of GO was obtained by dispersing a specific amount of nanosheets in THF at the ratio of 1:5 (mg:ml) using bath sonication for $15 \mathrm{~min}$. This colloidal solution was added to the PP solution and refluxed for $30 \mathrm{~min}$ at $110^{\circ} \mathrm{C}-120$ ${ }^{\circ} \mathrm{C}$ with vigorous stirring. Finally, the composite solutions where was cast and dried in vacuum oven for $5 \mathrm{~h}$ at $40{ }^{\circ} \mathrm{C}$. The same procedure was used for preparation of the RGO:PP nanocomposites. The dried nanocomposite materials were used for further analysis.

\section{Characterization techniques}

The functional groups on its surface of GO and RGO were determined by fourier transform infrared spectrophotometer (FTIR 8400 Shimadzu, Japan) with a resolution of $4 \mathrm{~cm}^{-1}$ over the frequency range $400-4000 \mathrm{~cm}^{-1}$. The specimens were prepared by mixing the ground samples with powdered potassium bromide and pressing the mixture under high pressure to obtain the pellet.

Crystalline nature was judged by X-ray powder diffractometer (XRD) Bruker, D8 ADVANCE, Germany, using monochromatic CuK $\alpha$ radiation $(\lambda=1.5406 \AA)$ at $40 \mathrm{kV}$ and $40 \mathrm{~mA}$. The scan rate was $5^{\circ} / \mathrm{min}$ between the angles $5-80^{\circ}$. Differential scanning calorimeter (DSC) was used to investigate melting temperature of nanocomposites using Shimadzu DSC 60 Tokyo, Japan. Temperature programming was done from 30 to $300^{\circ} \mathrm{C}$ at the heating rate of $10{ }^{\circ} \mathrm{C} / \mathrm{min}$ under an inert atmosphere in nitrogen with a flow rate of $50 \mathrm{ml} / \mathrm{min}$. TGA (Perkin Elmer TGA-4000, Switzerland) was used at heating rate of $10{ }^{\circ} \mathrm{C} / \mathrm{min}$ from room temperature to $750{ }^{\circ} \mathrm{C}$. The thermal stability 
of the nanocomposites was investigated over the range of 25- 500 ${ }^{\circ} \mathrm{C}$ at the heating rate of $10^{\circ} \mathrm{C} / \mathrm{min}$.

The tensile strength and modulus were determined as per ASTM D638 with a universal testing machine (Hi-Tech Industries \& Services, Model-UT2302, Mumbai) at a crosshead speed of $100 \mathrm{~mm} /$ min with load cell having capacity of 1 ton at room temperature.

Viscoelastic properties, i.e., storage modulus (E'), loss modulus(E"), and loss fact or ( $\tan \delta$ ) of PP and GNM based PP nanocomposites were measured on the DMA Q800 V20.24 Build 43 operating at a frequency of $1.0 \mathrm{~Hz}$, TA Instruments, USA. The amplitude and the static forces were $25 \mu \mathrm{g}$ and $0.01 \mathrm{~N}$, respectively. Data were collected from $-60{ }^{\circ} \mathrm{C}$ to $125{ }^{\circ} \mathrm{C}$ at a scanning rate of $3{ }^{\circ} \mathrm{C} / \mathrm{min}$. For dual cantilever mode, specimens in the shape of rectangular bars of nominal dimensions: $35 \mathrm{~mm} \times 10 \mathrm{~mm} \times 1 \mathrm{~mm}$ were prepared and used.

Surface morphology was observed using field emission scanning electron microscope (FE-SEM), HITACHI S-4800, operated at 5 to $15 \mathrm{Kv}$. Suspension was drop cast onto carbon tape and dried at room temperature. Prior to analysis, the sample was coated with gold to avoid degradation or burning due to high power. A transmission electron microscope (TEM) (Philips CM200, The Netherlands) was used to assess the morphology of composites at a resolution of $2.4 \AA$. The samples were dispersed in acetone and deposited on a copper grid before viewing under the microscope.

\section{Results and Discussion}

\section{FTIR analysis}

The exact chemical nature of GO and RGO was analyzed by FTIR spectroscopy and shown in Figure 1. The existence of several bands in the spectrum of GO confirmed presence of surface functionalities. The band at $3410 \mathrm{~cm}^{-1}$ corresponds stretching of the $-\mathrm{OH}$ bonds in hydroxyl groups. The bands between $1630 \mathrm{~cm}^{-1}$ and $1740 \mathrm{~cm}^{-1}$ assign to the stretching vibration of $\mathrm{C}=0$ in carboxyl groups, while the band at $1030 \mathrm{~cm}^{-1}$ corresponds to $\mathrm{C}=0$ in epoxide [33-36]. The FTIR spectrum of RGO is lack of noticeable bands, indicating that most of the oxygen-containing groups has been removed after the chemical reduction of GO. However, the noticeable band around $1480 \mathrm{~cm}^{-1}$ is observed and assigned to the aromatic $\mathrm{C}=\mathrm{C}$ of $\mathrm{sp}^{2}$ domain structure of RGO. FTIR spectrum of RGO shows a complete reduction of GO [37-39]. FTIR spectra of GOPP and RGOPP nanocomposites are depicted in Figure 2. Polypropylene shows characteristic vibrational peaks: $2930 \mathrm{~cm}^{-1}$ represents $-\mathrm{CH}_{3}$, $2910 \mathrm{~cm}^{-1}$ represents $-\mathrm{CH}_{3}$ aromatic group, $2870 \mathrm{~cm}^{-1}$ for $\mathrm{CH}_{2}$, and $2840 \mathrm{~cm}^{-1}$ represents $-\mathrm{CH}_{2}-\mathrm{CH}_{2} \cdot 1460 \mathrm{~cm}^{-1}$ and $1370 \mathrm{~cm}^{-1}$ represent $-\mathrm{CH}_{2}$ and $-\mathrm{CH}_{3}$ respectively $[40,41]$. FTIR vibrational peaks in composites do not show any massive change in case of GOPP, which is similar to neat PP; whereas intensity of peaks in RGOPP nanocomposite with an increase in the concentration of fillers, which indicates that polymer chains bind compactly with each other, and require maximum energy for vibration.

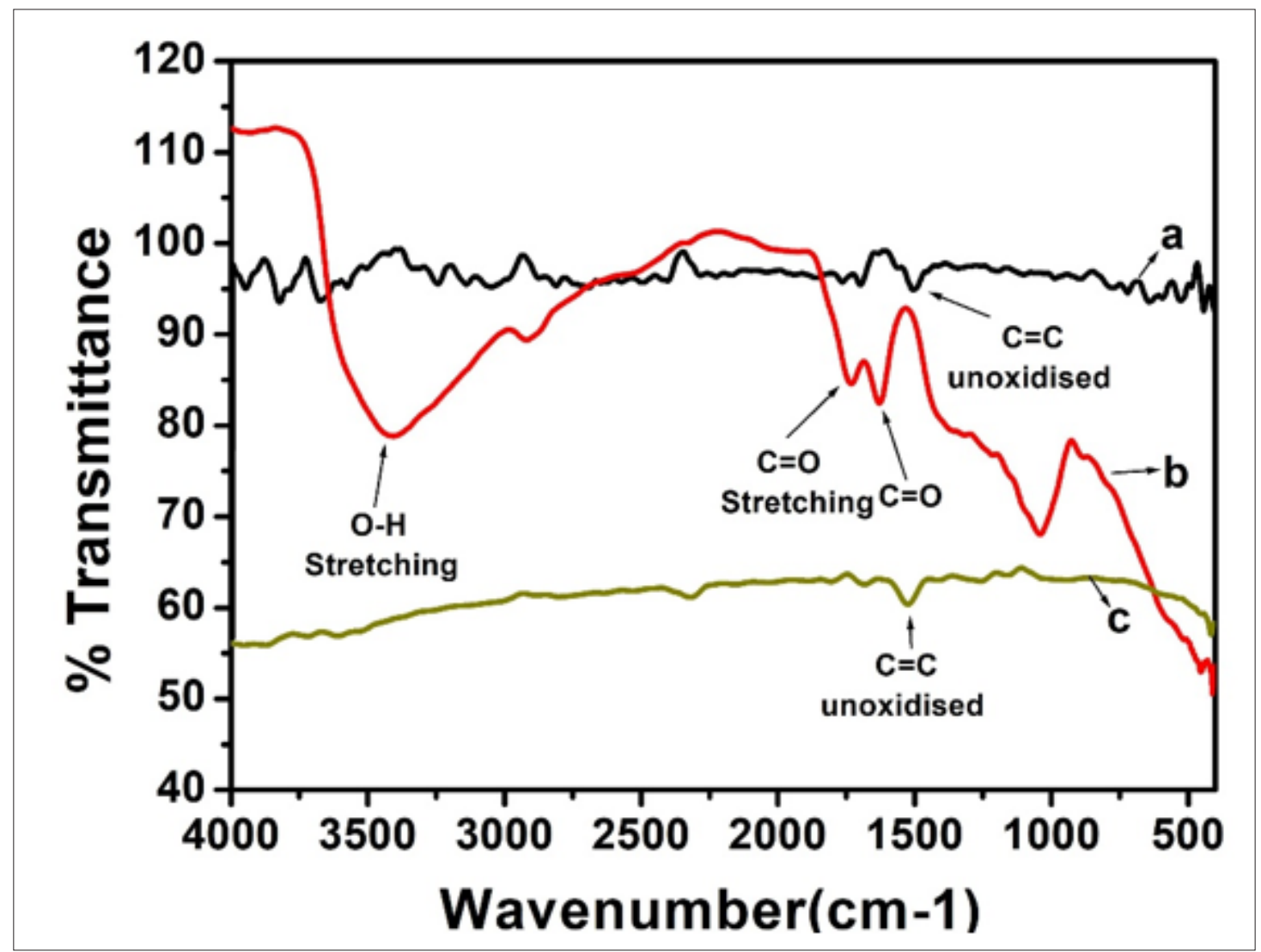

Figure 1: FTIR vibrational peaks of graphite powder, GO and RGO. 

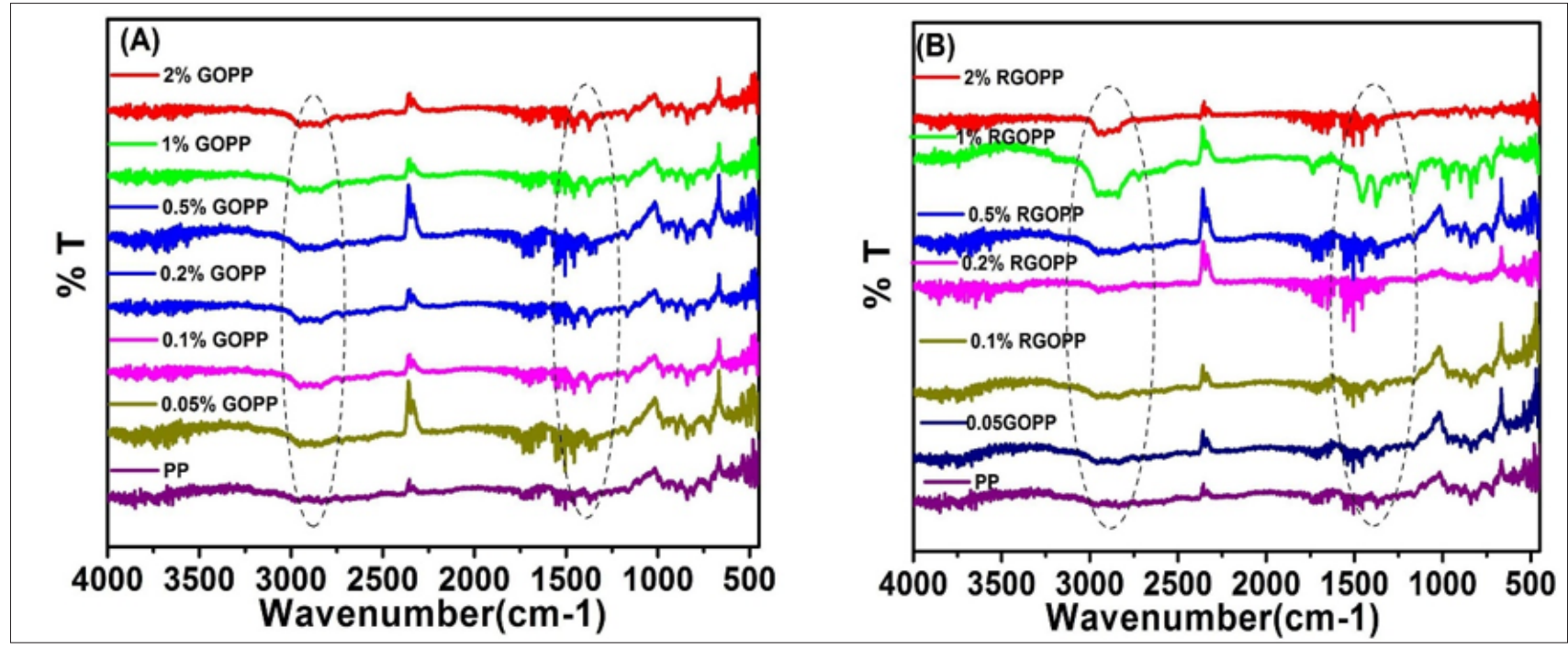

Figure 2: FTIR vibrational spectra of (A) GOPP, and (B) RGOPP nanocomposites.

\section{XRD analysis}

Figure 3 shows for pristine graphite, a sharp reflection was observed at $2 \theta=25.55^{\circ}$ corresponding to an interlayer spacing of $0.3 \mathrm{~nm}$. After oxidative treatment, graphite was converted in $\mathrm{GO}$; the diffraction peak shifted to $2 \theta=8.3^{\circ}$ with increased interlayer spacing up to $1.08 \mathrm{~nm}$ due to the intercalation of oxygen-containing groups [37]. In case of RGO, broad diffraction peak was observed at $2 \theta=23.60^{\circ}$ that corresponds to interlayer spacing of $0.37 \mathrm{~nm}$. The decrease in interlayer spacing for RGO was resulted by stacking of graphene sheets due to the strong Van der Waal forces between interlayers [38].

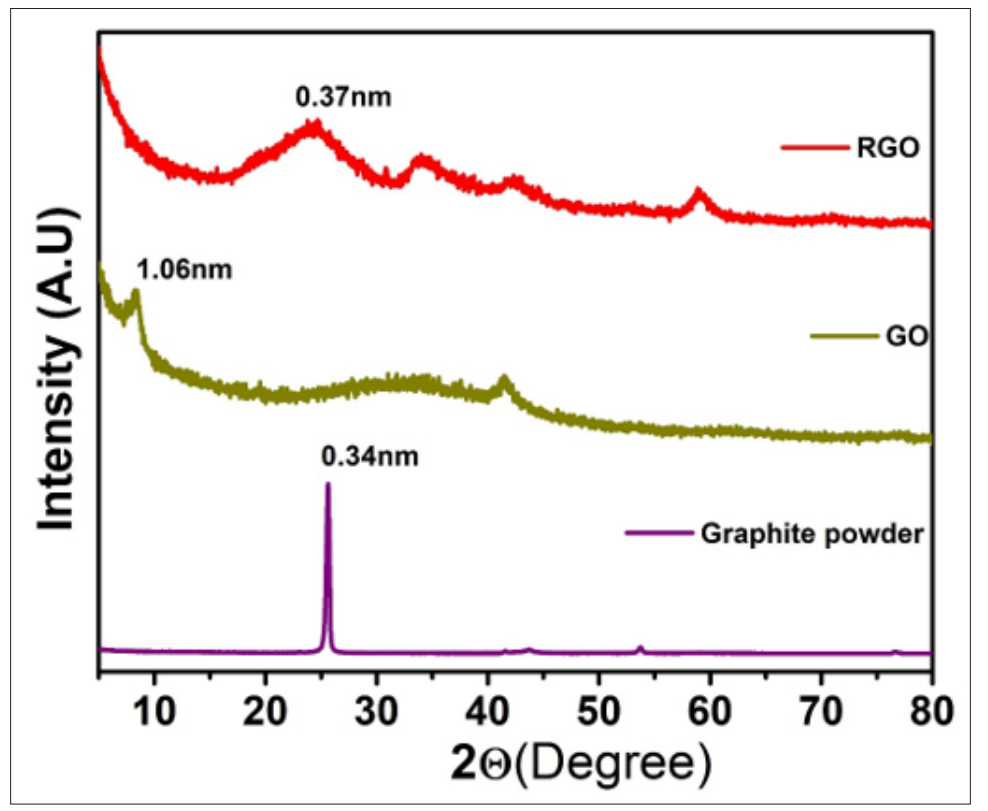

Figure 3: XRD pattern of graphite powder, GO and RGO.

XRD patterns of nanocomposites of GOPP and RGOPP show increase in the crystallinity of PP due to the graphitic nanofillers (Figure 4A \& 4B). The XRD profiles of the GOPP and RGOPP nanocomposites illustrate the broad diffraction peak corresponding to the uniform dispersion of GO and RGO nanosheets, In addition, the XRD patterns of GOPP and RGOPP composites show characteristic diffraction peaks at $2 \theta$ at $14.1^{\circ}, 16.1^{\circ}, 17.8^{\circ}, 21.1^{\circ}$ which correspond to $110,040,130,111$ crystalline planes of $\alpha$-form PP [41,42], respectively. The XRD patterns show an increase in the intensity of the peaks with an increase in the concentration of fillers as compared to virgin PP. This might be due to nanofillers, which may act as a nucleating agent that helps to provide a binding site for the polymer chains leading to increase in compactness of polymer chains. 

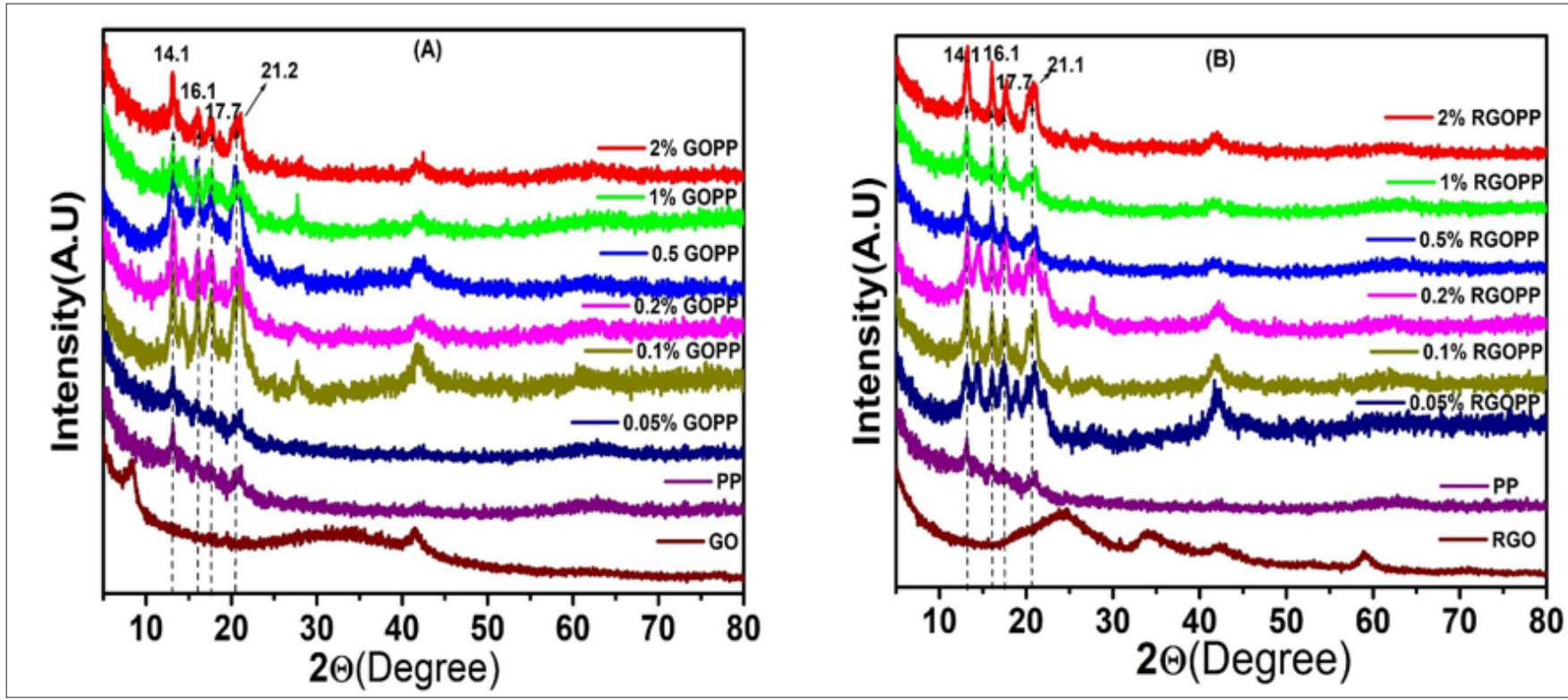

Figure 4: XRD pattern of (A), GOPP and (B), RGOPP nanocomposite.

\section{Thermal analysis}

DSC: Figure 5A \& 5B shows DSC curves of melting temperature (Tm) of GOPP and RGOPP nanocomposites. DSC curves give a clear indication of a slight, increase in melting temperature of PP matrix. In case of GOPP nanocomposites, the melting temperature profile was $165^{\circ} \mathrm{C}, 164{ }^{\circ} \mathrm{C}, 165^{\circ} \mathrm{C}$ and $164{ }^{\circ} \mathrm{C}$ with GO loadings of 0.2 wt. $\%$, 0.5wt.\%, $1 \mathrm{wt} . \%$ and $2 \mathrm{wt} . \%$ respectively. RGO incorporation in PP gives nearly the same results, except $167^{\circ} \mathrm{C}$ of melting temperature at 2 wt. \% loading of RGO which was recorded. While virgin PP melts at $163{ }^{\circ} \mathrm{C}$. The dispersed GO and RGO with PP results in increasing melting temperature. This is because the chains of PP were immobilized inside and onto the GO and RGO sheets that prevented the segmental motion of the polymer chains [43].
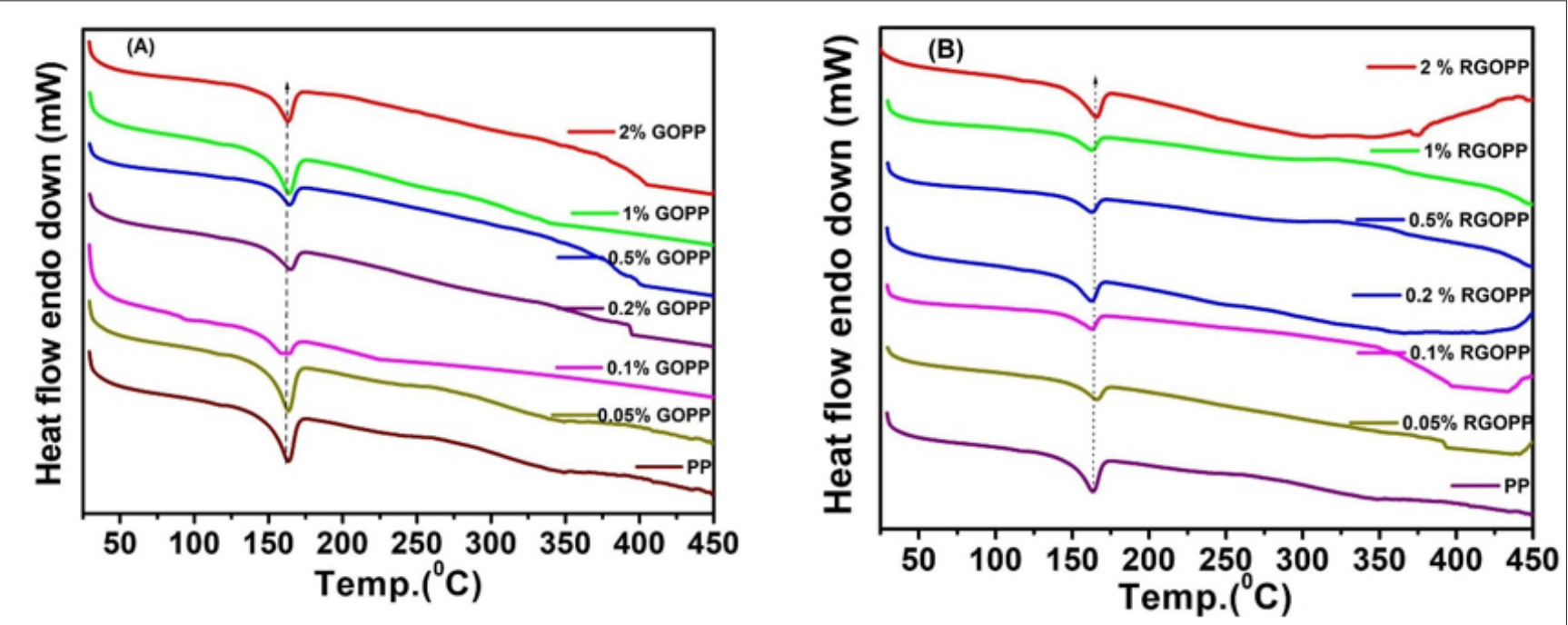

Figure 5: DSC curves of (A) GOPP and (B) RGOPP nanocomposite.

TGA: Primarily weight loss at $111^{\circ} \mathrm{C}$ for GO was due to evaporation of moisture coupled with it, which reflects its hydrophilic nature. A major weight loss at $173^{\circ} \mathrm{C}$ results due to the removal of oxygenated functionalities (Figure 6A). In case of RGO, thermal sta- bility is greater as compared to GO (Figure 6B). It shows just $30 \%$ weight loss up to $500^{\circ} \mathrm{C}$ because of the absence of functional groups on the surface of the sheet. A trend of improvement in thermal stability of the composites is same for both the systems (Table 1). 

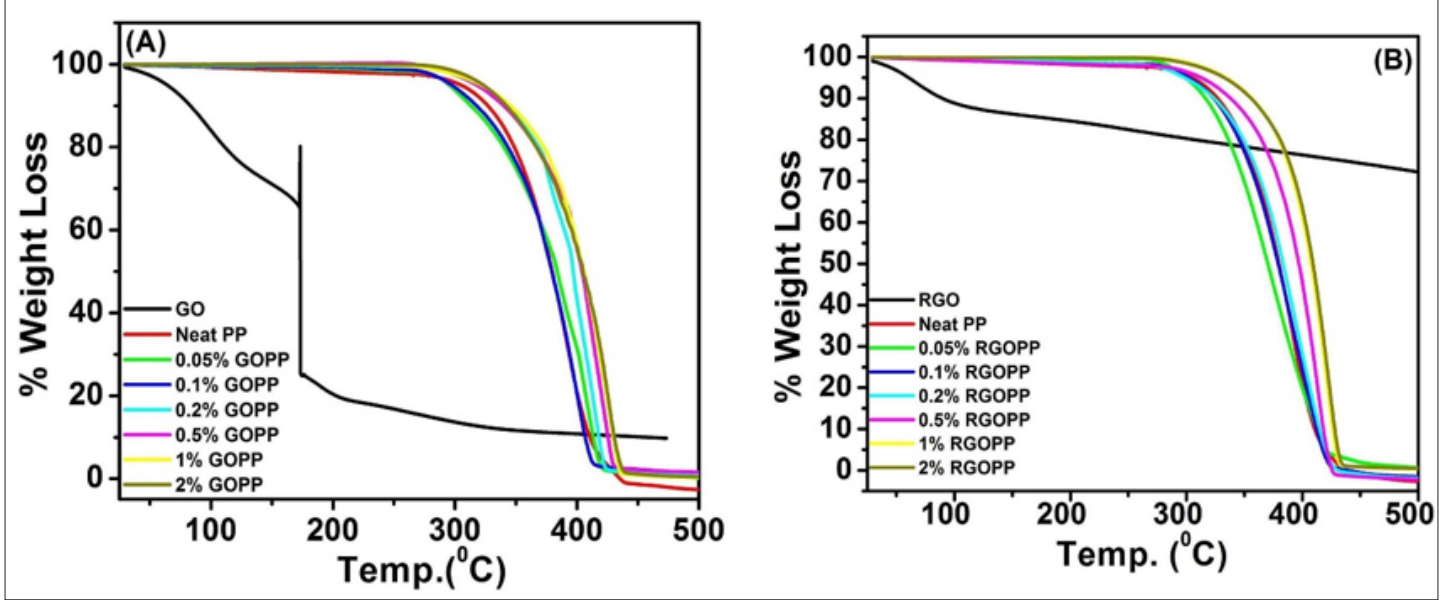

Figure 6: TGA curves of (A) GOPP and (B) RGOPP nanocomposite.

Table 1: Thermo gravimetric analysis of GOPP and RGOPP nanocomposite.

\begin{tabular}{|c|c|c|c|c|c|}
\hline Composite & $\begin{array}{c}20 \% \text { Weight Loss at } \\
\text { Temp. }\left({ }^{\circ} \mathrm{C}\right)\end{array}$ & $\begin{array}{l}80 \% \text { Weight Loss at Temp. } \\
\left({ }^{\circ} \mathrm{C}\right)\end{array}$ & Composite & $\begin{array}{l}20 \% \text { wt Loss at } \\
\text { Temp. }\left({ }^{\circ} \mathrm{C}\right)\end{array}$ & $\begin{array}{c}80 \% \text { Weight Loss at } \\
\text { Temp. }\left({ }^{\circ} \mathrm{C}\right)\end{array}$ \\
\hline PP & 348 & 400 & PP & 348 & 400 \\
\hline $0.05 w t \% G O P P$ & 339 & 407 & $0.05 w t \% R G O P P$ & 335 & 400 \\
\hline $0.1 \mathrm{wt} \%$ GOPP & 343 & 400 & $0.1 w t \%$ RGOPP & 334 & 403 \\
\hline $0.2 w t \%$ GOPP & 369 & 412 & $0.2 w t \%$ RGOPP & 348 & 406 \\
\hline $0.5 w t \%$ GOPP & 366 & 420 & $0.5 w t \%$ RGOPP & 364 & 413 \\
\hline 1 wt $\%$ GOPP & 373 & 424 & $1 w t \%$ RGOPP & 380 & 423 \\
\hline 2 wt $\%$ GOPP & 366 & 426 & $2 w t \%$ RGOPP & 380 & 423 \\
\hline
\end{tabular}

Improvement in thermal stability of PP by addition of GO and RGO was observed because they [32] may act as a binding agent which holds the chains of PP. When the temperature increases the mobility of polymer chain starts, and they get dislocated. The GO and RGO restrict the mobility of PP chains and show better thermal stability than the virgin PP $[43,44]$. Moreover, the GO and RGO sheets transfer the heat in greater extent so that polymer chains sustain for greater temperature.

\section{Mechanical study}

Tensile strength: Mechanical properties of PP nanocomposites were significantly improved as compared to virgin PP matrix. Tensile strength of GOPP was found to be $24 \mathrm{MPa}, 25 \mathrm{MPa}, 26 \mathrm{MPa}, 26 \mathrm{MPa}$, $26 \mathrm{MPa}$ and 28MPa at 0.05wt.\%, 0.1wt.\%, 0.2wt.\%, 0.5wt.\%, $1 \mathrm{wt} . \%$ and $2 \mathrm{wt} . \%$, respectively (Table $2 \mathrm{a}$ ), while $23 \mathrm{MPa}$ was recorded

Table 2a: Tensile strength (MPa) of GOPP nanocomposites. for virgin PP. In case of RGOPP, tensile strength was found to be $27 \mathrm{MPa}, 27 \mathrm{MPa}, 26 \mathrm{MPa}, 27 \mathrm{MPa}, 26 \mathrm{MPa}$ and $27 \mathrm{MPa}$ for $0.05 \mathrm{wt} . \%$, $0.1 w t . \%, 0.2 w t . \%, 0.5 w t . \%, 1$ wt. $\%$ and $2 w t . \%$ respectively (Table $3(\mathrm{a})$ ). Thus, highest tensile strength was obtained at $2 \mathrm{wt}$. \% of RGO loading in PP matrix.

Young's modulus: Like tensile strength, the PP matrix filled with 2 wt.\% of GO and RGO showed the maximum young modulus among the other proportions used. The young modulus values were observed as 28MPa for virgin PP, 44MPa for GOPP (Table 2(b)) and 42MPa for RGOPP (Table 3(b)) nanocomposites. Young modulus of prepared nanocomposites is higher as compared to virgin PP matrix. Uniform dispersion of GO and RGO in PP matrix results superior binding of polymer chains due to that it restricts deformation which leads to increase in modulus $[45,46]$.

\begin{tabular}{|c|c|c|c|c|c|c|}
\hline \multirow{2}{*}{ Nano Composite } & \multicolumn{3}{|c|}{ Tensile Strength (MPa) } & \multirow{2}{*}{ Mean Tensile Strength (MPa) } & \multirow{2}{*}{ Variance } & \multirow{2}{*}{ Standard Deviation $(\sigma)$} \\
\hline & 1 & 2 & 3 & & & \\
\hline PP & 19.67 & 22.67 & 25.48 & $22.60( \pm 5)$ & 8.44 & 2.9 \\
\hline 0.05wt.\%GOPP & 21.45 & 24.21 & 28.3 & $24.65( \pm 5)$ & 11.87 & 3.44 \\
\hline 0.1wt.\%GOPP & 22.88 & 26.25 & 29.6 & $26.24( \pm 5)$ & 11.28 & 3.36 \\
\hline $0.2 w t . \% G O P P$ & 26.15 & 28.1 & 24.1 & $26.11( \pm 5)$ & 4 & 2 \\
\hline 0.5wt.\%GOPP & 22.9 & 26.35 & 29.8 & $26.35( \pm 5)$ & 11.9 & 3.45 \\
\hline 1wt.\%GOPP & 30.4 & 20.49 & 25.43 & $25.44( \pm 5)$ & 24.55 & 4.95 \\
\hline 2wt.\%GOPP & 24.47 & 30.14 & 24.8 & $26.47( \pm 5)$ & 10.1289 & 3.18 \\
\hline
\end{tabular}


Table 2b: Young modulus (MPa) of GOPP nanocomposites.

\begin{tabular}{|c|c|c|c|c|c|c|}
\hline \multirow{2}{*}{ Nano Composite } & \multicolumn{3}{|c|}{ Young modulus (MPa) } & \multirow{2}{*}{ Mean Young Modulus (MPa) } & \multirow{2}{*}{ Variance } & \multirow{2}{*}{ Standard Deviation $(\sigma)$} \\
\hline & 1 & 2 & 3 & & & \\
\hline PP & 27.86 & 26.48 & 29.24 & $27.86( \pm 3)$ & 1.9 & 1.38 \\
\hline 0.05wt.\%GOPP & 27.12 & 28.49 & 25.75 & $27.12( \pm 3)$ & 1.8 & 1.37 \\
\hline 0.1wt.\%GOPP & 25.66 & 29.11 & 22.31 & $25.66( \pm 3)$ & 11.56 & 3.4 \\
\hline $0.2 \mathrm{wt} . \% \mathrm{GOPP}$ & 31.47 & 32.78 & 30.16 & $31.47( \pm 3)$ & 1.71 & 1.31 \\
\hline 0.5wt.\%GOPP & 34.82 & 32.46 & 37.18 & $34.82( \pm 3)$ & 5.56 & 2.36 \\
\hline 1wt.\%GOPP & 43.54 & 44.43 & 42.65 & $43.54( \pm 3)$ & 0.7 & 0.89 \\
\hline 2wt.\%GOPP & 44.01 & 46.11 & 41.91 & $44.01( \pm 3)$ & 4.41 & 2.1 \\
\hline
\end{tabular}

Table 3a: Tensile strength (MPa) of RGOPP nanocomposites.

\begin{tabular}{|c|c|c|c|c|c|c|}
\hline \multirow{2}{*}{ Nano Composite } & \multicolumn{3}{|c|}{ Tensile Strength (MPa) } & \multirow{2}{*}{ Mean Young Modulus (MPa) } & \multirow{2}{*}{ Variance } & \multirow{2}{*}{ Standard Deviation $(\sigma)$} \\
\hline & 1 & 2 & 3 & & & \\
\hline PP & 19.67 & 22.67 & 25.48 & $22.60( \pm 5)$ & 8.44 & 2.9 \\
\hline 0.05wt.\%RGOPP & 26.66 & 24.68 & 28.64 & $26.66( \pm 5)$ & 3.92 & 1.98 \\
\hline 0.1wt.\%RGOPP & 26.66 & 29.55 & 23.77 & $26.66( \pm 5)$ & 8.35 & 2.89 \\
\hline 0.2wt.\%RGOPP & 26.35 & 27.12 & 25.59 & $26.35( \pm 5)$ & 0.58 & 0.76 \\
\hline 0.5wt.\%RGOPP & 26.76 & 27.11 & 26.41 & $26.76( \pm 5)$ & 0.12 & 0.35 \\
\hline 1wt.\%RGOPP & 27.17 & 20.14 & 34.2 & $27.17( \pm 5)$ & 49.42 & 7.03 \\
\hline 2wt.\%RGOPP & 25.53 & 20.14 & 28.6 & $25.53( \pm 5)$ & 9.42 & 3.07 \\
\hline
\end{tabular}

Table 3b: Young modulus (MPa) of RGOPP nanocomposites.

\begin{tabular}{|c|c|c|c|c|c|c|}
\hline \multirow{2}{*}{ Nano Composite } & \multicolumn{3}{|c|}{ Young Modulus (MPa) } & \multirow{2}{*}{ Mean Young Modulus (MPa) } & \multirow{2}{*}{ Variance } & \multirow{2}{*}{ Standard Deviation $(\sigma)$} \\
\hline & 1 & 2 & 3 & & & \\
\hline PP & 27.86 & 26.48 & 29.24 & $27.86( \pm 3)$ & 1.9 & 1.38 \\
\hline 0.05wt.\%RGOPP & 29.46 & 24.59 & 34.33 & $29.46( \pm 3)$ & 23.71 & 4.87 \\
\hline 0.1wt.\%RGOPP & 29.88 & 30.43 & 30.98 & $30.43( \pm 3)$ & 0.3 & 0.55 \\
\hline 0.2wt.\%RGOPP & 30.5 & 34.26 & 32.38 & $32.38( \pm 3)$ & 3.53 & 1.88 \\
\hline 0.5wt.\%RGOPP & 34.11 & 32.54 & 30.97 & $32.54( \pm 3)$ & 2.4 & 1.57 \\
\hline 1wt.\%RGOPP & 32.72 & 31.1 & 34.34 & $32.72( \pm 3)$ & 2.6 & 1.62 \\
\hline 2wt.\%RGOPP & 42.98 & 41.9 & 40.82 & $41.90( \pm 3)$ & 1.1 & 1.08 \\
\hline
\end{tabular}

\section{Viscoelastic properties}

Dynamic mechanical measurements provide information about Viscoelastic properties of material with temperature.

Storage modulus: Figure 7A shows the storage modulus of PP, GOPP and RGOPP nanocomposites. The storage modulus dramatically increased with the addition of GO and RGO as fillers in PP matrix. An increase in the temperature towards melting point shows the transition where the peak intensity was lower as compared to that of virgin PP. The storage modulus at $-60{ }^{\circ} \mathrm{C}$ was found to be $8728 \mathrm{MPa}, 9201 \mathrm{MPa}$ and $9036 \mathrm{MPa}$ for PP, $1 \mathrm{wt} . \% \mathrm{GOPP}$, and RGOPP nanocomposites respectively of respectively. Results showed that nanocomposites were storing the maximum energy as compared to virgin PP [25].

Loss modulus: Loss modulus is depicted in Loss modulus $\left(E^{\prime \prime}\right)$ represents the energy dissipated in one cycle as heat during deformation of the PP, GOPP and RGOPP nanocomposites (Figure
7B). PP exhibits three transition peaks $(\alpha, \beta$, and $\Upsilon)$ at different temperatures within the investigated temperature range $\left(6{ }^{\circ} \mathrm{C}\right.$ to $\left.125^{\circ} \mathrm{C}\right)[47,48]$. The $\Upsilon$-transition near to $100{ }^{\circ} \mathrm{C}$ represents the relaxation of amorphous propylene segments of the PP chain. The $\beta$-relaxation is barely visible as a shoulder on the $\alpha$-relaxation. The $\beta$-relaxation is strongly dependent on density of PP. The variations in E" for the virgin PP, GOPP and RGOPP nanocomposites are functions of temperature. The $\beta$-transition is due to the $\mathrm{Tg}$ in the range of -10 ${ }^{\circ} \mathrm{C}$ to $10{ }^{\circ} \mathrm{C}$ which is associated with the motion of the long chain segments in the amorphous region of the PP [21]. In this study, the $\beta$-transition peak of the matrix was observed at around $7{ }^{\circ} \mathrm{C}$. The $\beta$ relaxation is referred to as the temperature at the maximum value of loss modulus. As per results observed RGOPP and PP showed the same intensity (200MPa) of the peak at $\beta$ relaxation, but GOPP shows significant changes in value (225MPa), relative to virgin PP which indicates that GOPP decreases the mobility of polymer chains in greater extent due to and maximum energy dissipation compared to neat PP [49]. 

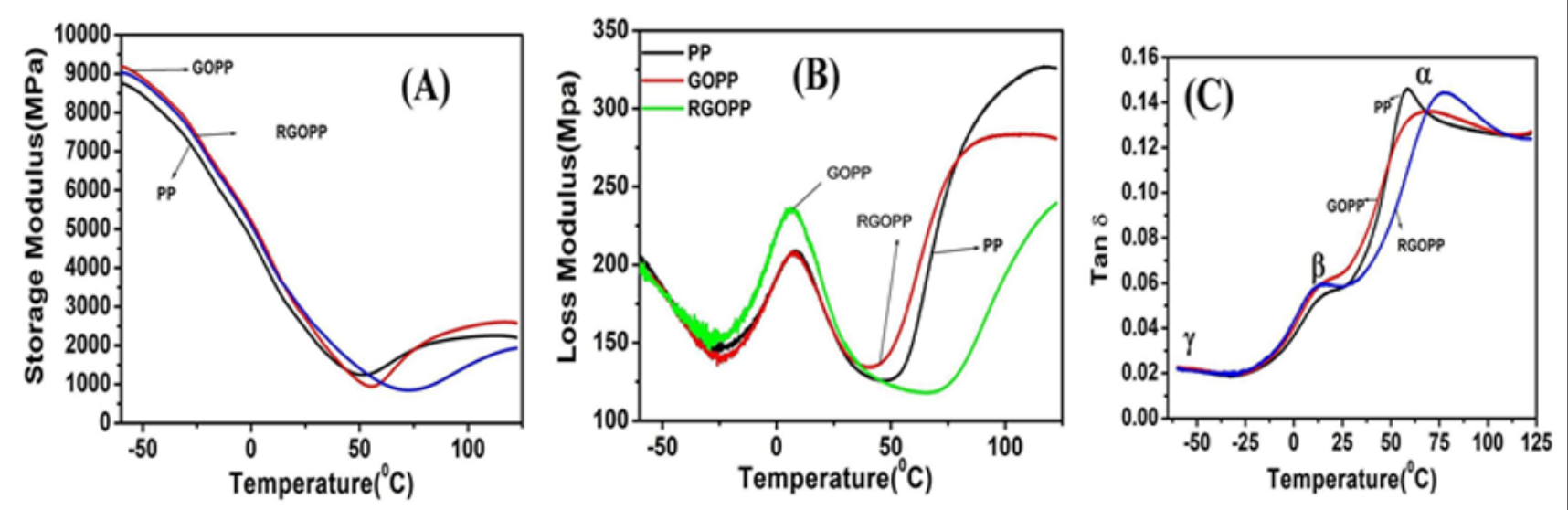

Figure 7: Storage modulus (A), Loss modulus (B) and Tan delta (C) of PP, GOPP and RGOPP nanocomposite.

Loss tangent: Loss tangent $(\tan \delta$ ), ratio of the loss modulus to the storage modulus, is measured as the mechanical loss or damping factor $(\tan \delta$ ) [50]. The variations of $\tan \delta$ as a function of temperature are represented in Figure 8C. The transition region as indicated by damping maxima is usually known as the $\beta$-transition. The transformations are $\alpha, \beta$ and $\Upsilon$ in decreasing order of the temperature, as labeled in figure. The $\Upsilon$ mechanism overlaps the $\beta$ process and has attributed to local motion in the amorphous phase of PP. The $\beta$ relaxation is generalized motion in amorphous regions during the glass transition [47]. The transition at higher temperature is attributed to the mobility within crystalline region [25]. $\beta$ relaxation slightly moves towards higher temperature (12 ${ }^{\circ} \mathrm{C}, 13{ }^{\circ} \mathrm{C}$ and $15{ }^{\circ} \mathrm{C}$ for PP, GOPP, and RGOPP respectively) because GO and RGO form a compact and rigid structure of PP. Changes in the mobility within crystallites seem to be more important, and the mechanism accordingly shifts to higher temperature $57{ }^{\circ} \mathrm{C}, 67^{\circ} \mathrm{C}$ and $77^{\circ} \mathrm{C}$ for PP, GOPP, and RGOPP respectively. The peak intensity of nanocomposites was in order $\mathrm{PP}>\mathrm{RGOPP}>\mathrm{GOPP}$. Thus, the GOPP is more rigid than the RGOPP followed by virgin PP.

\section{FESEM analysis}

Figure 8 shows images of GO and GOPP nanocomposites where image (a) shows thin layer and separate GO sheets indicating the exfoliation graphite. EDS analysis shows elemental detection, a complete idea about the presence of oxygen functionalities on the surface of the GO sheet (Table 4). Image (b) represents neat PP; it shows that some of the pores are available on the surface and rough in nature. Images (c), (d), (e), (f), (g) and (h) represent the GO/PP nanocomposites with different wt.\% proportions i.e $0.05 w t . \%$, 0.1wt.\%, 2wt.\%, 0.5 wt.\%, 1 wt.\% and $2 w t . \%$ respectively. All images show that there is a uniform dispersion of GO sheets in PP. GO sheets are clearly observed on the surface of nanocomposites. As increases the concentration of GO in PP, the appearance of GO sheets becomes more uniform in nature. Thus, the concentrations of GO and RGO directly affect the compactness of PP chains in the nanocomposites. Figure 9A shows the morphology of RGO in which RGO sheets are in shrinking form, due to the removal of functional groups which leads to reduce the interlayer distance. Images (c), (d), (e), (f), (g) and (h) represent the RGOPP nanocomposites with proportion $0.05 w t . \%, 0.1 w t . \%, 0.2 w t . \%, 0.5 w t . \%, 1 w t . \%$ and $2 w t . \%$ RGO respectively. The images give a clear idea of uniform dispersion of RGO in PP matrix. The appearance of RGO becomes more visible on increasing the concentration of RGO (Figure 9B) in nanocomposites. Virgin PP shows some pores on the surface, while surface become smooth and compact after addition of GO and RGO fillers.

Table 4: Elemental analysis (EDS) of GO and RGO.

\begin{tabular}{|c|c|c|c|c|c|c|}
\hline Material & Element & Series & Unn. C [wt.\%] & Norm. C [wt.\%] & Atom. C [at.\%] & Error (1 Sigma) [wt.\%] \\
\hline \multirow{3}{*}{ GO } & $\mathrm{C}$ & K series & 61.98 & 62.45 & 68.38 & 7.64 \\
\hline & $\mathrm{N}$ & K series & 6.45 & 6.5 & 6.11 & 1.8 \\
\hline & 0 & K series & 30.81 & 31.04 & 25.52 & 4.58 \\
\hline \multirow{5}{*}{ RGO } & $\mathrm{C}$ & K series & 37.67 & 69.09 & 85.6 & 5.34 \\
\hline & 0 & K series & 7.15 & 13.12 & 12.2 & 1.65 \\
\hline & $\mathrm{Al}$ & K series & 0.54 & 0.99 & 0.55 & 0.07 \\
\hline & $\mathrm{Ca}$ & K series & 0.7 & 1.28 & 0.47 & 0.09 \\
\hline & $\mathrm{Au}$ & M series & 8.46 & 15.52 & 1.17 & 0.41 \\
\hline
\end{tabular}




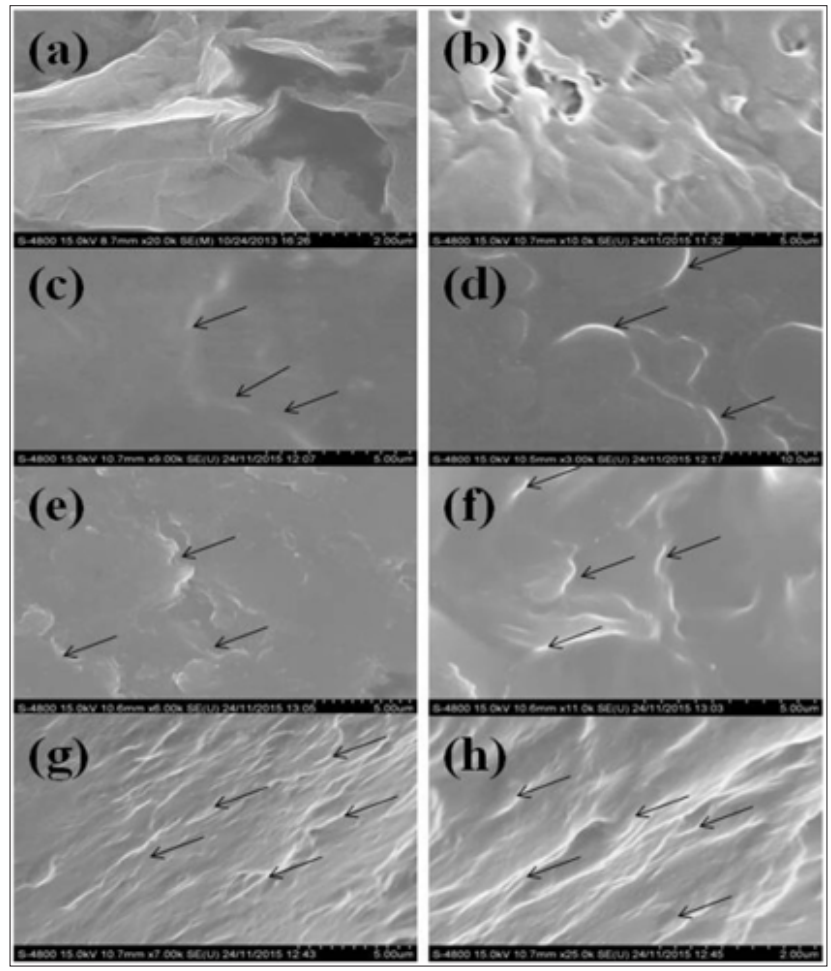

Figure 8: FESEM analysis of GOPP composite where(a)GO, (b)PP, (c)0.05wt\%GOPP, (d)0.1wt\%GOPP (e) $0.2 \mathrm{wt} \% \mathrm{GOPP},(\mathrm{f}) 0.5 \mathrm{wt} \% \mathrm{GOPP}$, (g) $1 \mathrm{wt} \% \mathrm{GOPP}$ and (h) $2 \mathrm{wt} \% \mathrm{GOPP}$.

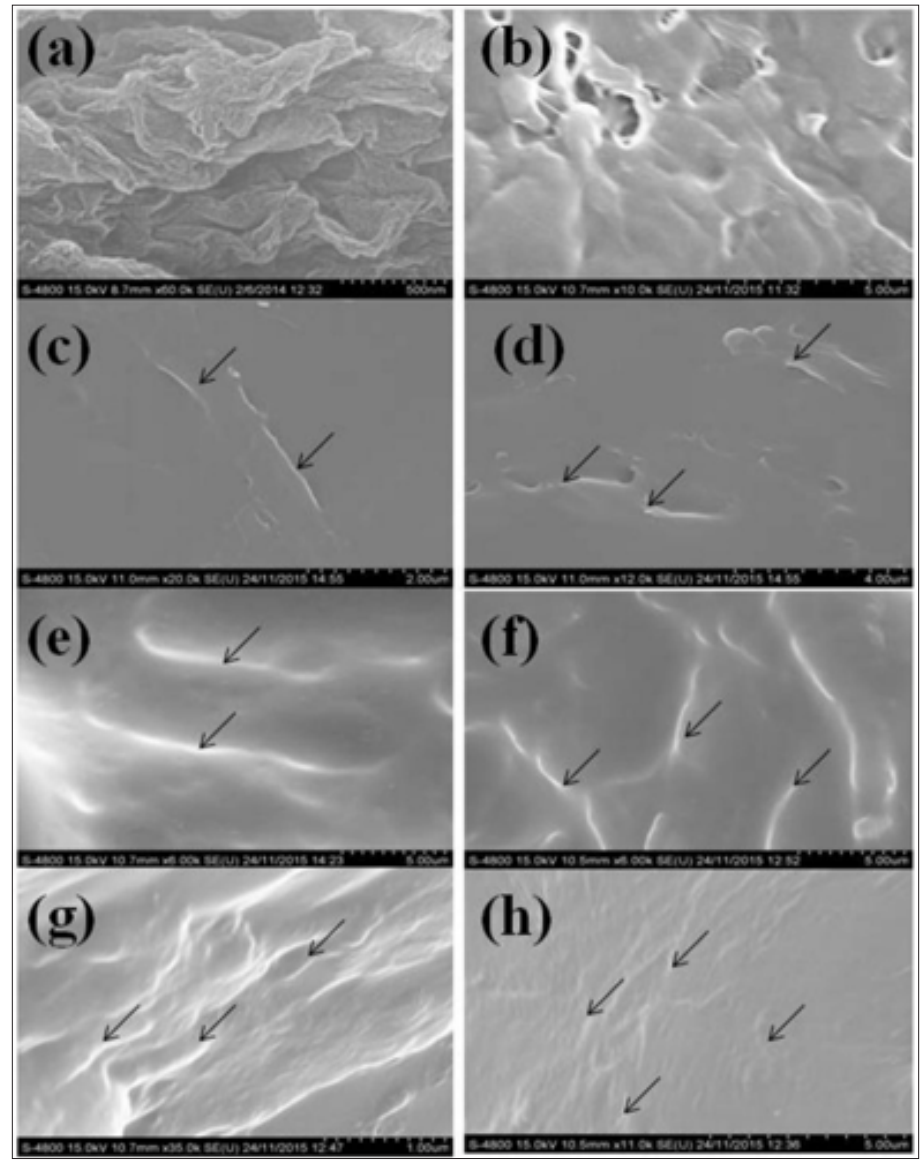

Figure 9: FESEM analysis of RGOPP composite where(a)RGO, (b)PP, (c)0.05wt\%RGOPP, (d)0.1wt\%RGOPP (e) $0.2 w t \% R G O P P,(f) 0.5 w t \% R G O P P,(g) 1 w t \% R G O P P$ and (h) $2 w t \% R G O P P$. 


\section{TEM analysis}

Figure 10 shows TEM images of GO, RGO and GOPP, and RGOPP nanocomposites. Image (a) represents GO which looks ultra-thin single sheet just because of the exfoliation of graphite sheets. TEM images give support to XRD data which confirm the exfoliation. The shrinking of RGO nanosheets was observed due to the reduction of functional groups. In case of GOPP nanocomposites, GO uniformly dispersed in the PP chains, clearly visualizing the single sheet in PP matrix. While RGO sheets are well dispersed but show some agglomeration rather shrinkage of sheets in the polymer matrix, which reduce the effective surface area of the RGO sheets.

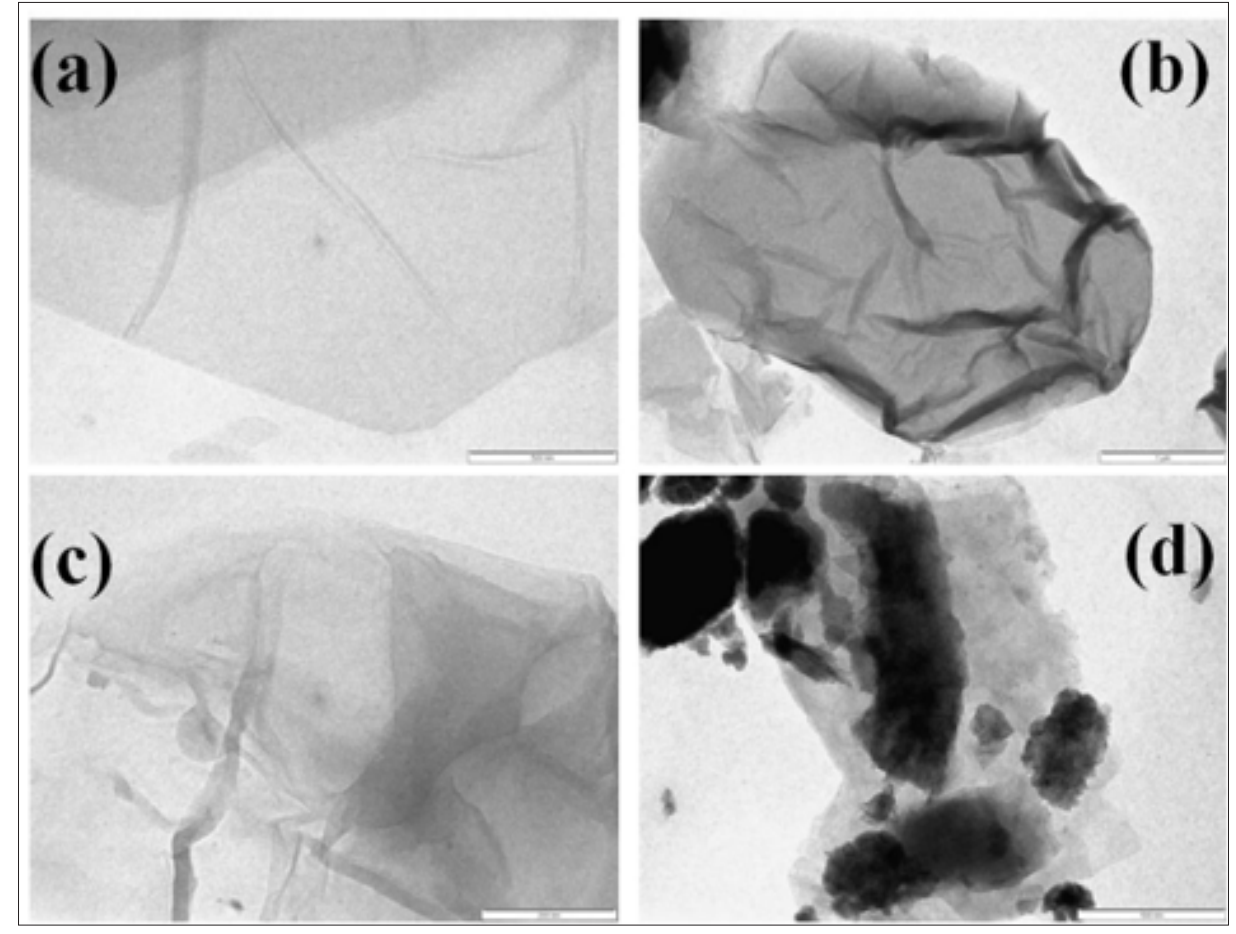

Figure 10: TEM analysis of (a) GO, (b)RGO, (c) GOPP and (d)RGOPP nanocomposite.

\section{Conclusion}

GO, and RGO successfully loaded in the PP matrix by solution blending. Prepared GOPP and RGOPP nanocomposite were characterized by FT-IR, XRD, DSC, TGA, DMA, FESEM, and TEM. Significant improvements were observed on thermal stability (above $23-26{ }^{\circ} \mathrm{C}$ ); Young modulus by $57 \%$ in GOPP and $50 \%$ in RGOPP; Tensile strength up to $10 \%$. Viscoelastic study of nanocomposites showed improvement of storage modulus by addition of GO and RGO. Based on results, we conclude that GO shows better improvements than RGO due to greater effective surface area of GO than the RGO as a filler in PP.

\section{Acknowledgment}

CRM is thankful to Technical Education Quality Improvement Program (TEQIP-II) for providing financial assistance to carry out this research work. SM is thankful to the University Grants Commission, New Delhi for awarding UGC BSR Fellowship. The authors also thank SAIF IIT-Powai, SAIF-UICT, NMU Jalgaon for characterizations of samples.

\section{References}

1. Novoselov KS, Geim AK, Morozov SV, Jiang D, Zhang Y, et al. (2004) Electric field effect in atomically thin carbon films. Science 306(5696): 666-669.
2. Lee C, Wei X, Kysar JW, Hone J (2008) Measurement of the elastic properties and intrinsic strength of monolayer graphene. Science 321(5887): 385-388.

3. Bharadiya P, Mishra S (2019) Enriched mechanical properties of epoxy/ coir fiber composites with graphene oxide. Research \& Development in Material Science 10(5): 1215-1222.

4. Cardinali M, Valentini L, Kennya JM, Mutlay I (2012) Graphene based composites prepared through exfoliation of graphite platelets in methyl methacrylate/poly(methylmethacrylate). Polym Int 61:1079-1083.

5. Sheshmani S, Amini R (2013) Preparation and characterization of graphene-based nanocomposite materials. Carbohydr Polym 95(1): 348-359.

6. Hansora DP, Mishra S (2017) Graphene nanomaterials: fabrication, properties, and applications. CRC Press, USA

7. Pasquini N (2005) Polypropylene, Hanser Gardner Publications, USA

8. Lomate G, Dandi B, Mishra S (2018) Development of antimicrobial LDPE/Cu nanocomposite food packaging film for extended shelf life of peda. Food Packaging and Shelf Life 16: 211-219

9. Ataeefard M, Moradian S (2012) Preparing dyeable PP fiber nanocomposites using the special cubic mixture experimental design. The Journal of the Textile Institute 103(11): 1169-1182.

10. Garcos JM, Moll DJ, Bicerano J, Fibiger R, McLeod DG (2000) Polymeric nanocomposites for automotive applications. Adv Mater 12(23): 18351839. 
11. Hong CH, Lee YB, Bae JW, Jho JY, Nam BU, et al. (2005) Preparation and mechanical properties of polypropylene/clay nanocomposites for automotive parts application. Journal of Applied Polymer Science 98(1): 427-433.

12. Kausar A, Rafique I, Muhammad B (2017) Aerospace application of polymer nanocomposite with carbon nanotube, graphite, graphene oxide and nanoclay. Polymer-Plastics Technology and Engineering 56(13): 1438-1456.

13. Riccioa A, Raimondo A, Sellitto A, Acanfora V, Zarrelli M (2016) Multifunctional polypropylene core for aerospace sandwich composite panels. Procedia Engineering 167: 64-70.

14. Onder E, Sarier N, Ersoy MS (2012) The manufacturing of polyamide and polypropylene organoclay nanocomposite filaments and their suitability for textile applications. Thermochimica Acta 543: 37-58.

15. Khan M, Ali M (2018) Effectiveness of hair and wave polypropylene fibers for concrete roads. Construction and Building Materials 166: 581591.

16. Hiremath PN, Yaragal SC (2018) Performance evaluation of reactive powder concrete with polypropylene fibers at elevated temperatures. Construction and Building Materials 169: 499-512.

17. Zhang J, Jiang D, Wilkie C (2005) Polyethylene and polypropylene nanocomposites based upon an oligomerically modified clay. Thermochimica Acta 430(1-2): 107-113.

18. Shimpi NG, Shirole S, Suryawanshi Y, Mishra S (2015) Optimized synthesis of ntio 2 using murraya koenigii leaf extract and its application in ipp-epdm blends. Ad Poly Tech 36(2): 160-167.

19. Shimpi N, Shirole S, Mishra S (2015) Polypropylene $/ \mathrm{nTiO}_{2}$ nanocomposites using melt mixing and its investigation on mechanical and thermal properties. Polymer Composites 38(7): 1273-1279.

20. Shimpi NG, Mali A, Sonawane H, Mishra S (2014) Effect of $\mathrm{nBaCO}_{3}$ on mechanical, thermal and morphological properties of isotactic PP-EPDM blend. Polym Bull 71: 2067-2080.

21. Mishra S, Chatterjee A, Singh R (2011) Novel synthesis of nanocalcium carbonate $\left(\mathrm{CaCO}_{3}\right) /$ polystyrene (PS) core-shell nanoparticles by atomized micro emulsion technique and its effect on properties of polypropylene (PP) composites. Polymers for Advanced Technologies 22(12): 2571-2582.

22. Mishra S, Chatterjee A (2011) Effect of nano-polystyrene (nPS) on thermal, rheological and mechanical properties of polypropylene (PP). Polymers for Advanced Tech 22(12): 1547-1554.

23. Bari P, Lanjewar S, Hansora DP, Mishra S (2016) Influence of the coupling agent and graphene oxide on the thermal and mechanical behavior of tea dust-polypropylene composites. Journal of Applied Polymer Science 133(4): 42927

24. Feng Qiu, Yongbo Hao, Xiaoyun Li, Wang B, Ming Wang (2015) Functionalized graphene sheets filled isotactic polypropylene nanocomposites. Composite part 71: 175-183.

25. Milani MA, González D, Quijada R, Basso N, Cerrada M, et al. (2013) Polypropylene/graphene nanosheet nanocomposites by in situ polymerization: Synthesis, characterization and fundamental properties. Composites Science and Technology 84: 1-7.

26. Hemmati M, Rahimi GH, Kaganj AB, Sepehri S, Rashidi AM (2008) Rheological and mechanical characterization of multi-walled carbon nanotubes/polypropylene nanocomposites. Journal of Macromolecular Science Physics 47(6): 1176-1187.

27. Ryu S, Shanmugharaj AM (2014) Influence of long-chain alkylaminemodified graphene oxide on the crystallization, mechanical and electrical properties of isotactic polypropylene nanocomposites. Chemical Engineering Journal 244: 552-560

28. Yang J, Huang Y, Lv Y, Zhao P, Yang Q, et al. (2013) The intrinsic thermaloxidative stabilization effect of chemically reduced graphene oxide on polypropylene. J Mater Chem 1: 11184.
29. Sanchez VS, Zapata DAG, Martinez CJG, Mendez NJ, Ramosde VLF, et al. (2018) Influence of functionalized polypropylene on polypropylene/ graphene oxide nanocomposite properties. Polymer Composites 39(4): 1361-1369.

30. Wang CC, Zhao YY, Ge HY, Qian RS (2018) Enhanced mechanical and thermal properties of short carbon fiber reinforced polypropylene composites by graphene oxide. Polym Compos 39: 405-413.

31. Miao M, Wei C, Wang Y, Qian Y (2018) Effect of compatibilizer on the interface bonding of graphene oxide/polypropylene composite fibers. Polymers 10: 1283.

32. Mantia FP, Ceraulo M, Mistretta MC, Botta L (2018) Effect of the elongational flow on morphology and properties of polypropylene/ graphene nanoplatelets nanocomposites. Polym Testing.

33. Marcano DC, Kosynkin DV, Berlin JM, Sinitskii A, Sun Z, et al. (2010) Improved synthesis of graphene oxide. ACS Nano 4: 4806-4814.

34. Bari P, Khan S, Njuguna J, Mishra S (2017) Elaboration of properties of graphene oxide reinforced epoxy nanocomposites. Int J Plast Technol 21(1): 194-208.

35. Jain R, Mishra S (2016) Electrical and electrochemical properties of graphene modulated through surface functionalization. RSC Advance 6: 27404.

36. Watson G, Starost K, Bari P, Faisal N, Mishra S, et al. (2017) Tensile and flexural properties of hybrid graphene oxide/epoxy carbon fibre reinforced composites. IOP Conference Series: Materials Science and Engineering 195(1): 012009

37. Mahajan C, Chaudhari P, Mishra S (2018) RGO-MWCNT-ZnO based polypyrrole nanocomposite for ammonia gas sensing. Journal of Materials Science: Materials in Electronics 29(10): 8039-8048.

38. Shen J, Li T, Long Y, Shi M, Li N, et al. (2012) One-step solid state preparation of reduced graphene oxide. Carbon 50(6): 2134-2140.

39. Hansora DP, Shimpi NG, Mishra S (2015) Graphite to graphene via graphene oxide: an overview on synthesis, properties, and applications 67(12): 2855-2868.

40. Park SM, Kim DS (2016) Preparation and physical properties of polypropylene nanocomposites with dodecylated graphene nanoplatelets. Composite Interfaces 24(4): 335-345.

41. Dai J, Liu X, Yang J, Zhang N, Huang T, et al. (2014) Stretching induces pore formation in the $\beta$-nucleated polypropylene/graphene oxide composite. Composites Science and Technology 99: 59-66.

42. Wang D, Zhang X, Zha JW, Zhao J, Dang ZM, et al. (2013) Dielectric properties of reduced graphene oxide/polypropylene composites with ultralow percolation threshold. Polymer 54(7): 1916-1922.

43. Zhao S, Chen F, Huang Y, Dong J, Han C (2014) Crystallization behaviors in the isotactic polypropylene/graphene composites. Polymer 55: 41254135.

44. Polshchikov SV, Nedorezova PM, Klyamkina AN, Krashenninikov VG, Aladyshev AM, et al. (2013) Composite materials based on graphene nanoplatelets and polypropylene derived via in situ polymerization. Nanotechnologies in Russia 8(1-2): 69-80.

45. Chen Y, Yin Q, Zhang X, Xue X, Jia H (2018) The crystallization behaviors and rheological properties of polypropylene/graphene nanocomposites: The role of surface structure of reduced graphene oxide, Thermochimica Acta 661: 124-136.

46. Triantou M, Todorova N, Giannakopoulou T, Vaimakis T, Trapalis C (2017) Mechanical performance of re-extruded and aged graphene/ polypropylene nanocomposites. Polym Int 66(12): 1716-1724.

47. Yun YS, Bae YH, Kim DH, Lee JY, Chin IJ, et al. (2011) Reinforcing effects of adding alkylated graphene oxide to polypropylene Carbon 49(11): 3553-3559.

48. Samal SK, Mohanty S, Nayak SK (2009) Polypropylene-bamboo/ glass fiber hybrid composites: fabrication and analysis of mechanical, 
morphological, thermal, and dynamic mechanical behavior. J Reinf Plast Compos 28(22): 2729-2747.

49. Yang J, Lin Y, Wang J, Lai M, Li J, et al. (2005) Morphology, thermal stability, and dynamic mechanical properties of atactic polypropylene/ carbon nanotube composites. Journal of Applied Polymer Science 98(3): 1087-109.
50. Rahman NA, Hassan A, Lafia-Araga R, Hornsby PR (2012) Polypropylene/ glass fiber/nanoclay hybrid composites: morphological, thermal, dynamic mechanical and impact behaviors. J Reinf Plast Compos 31(18): 1247-1250.

For possible submissions Click below:

Submit Article 